\title{
Structural characterization of the lid of lipase 2 of Pseudomonas alcaligenes
}

\author{
Elena Lizbeth Garcia-Villegas ${ }^{1}$, Enrique Rudiño-Piñera², Liliana Pardo² \\ ${ }^{1}$ Centro de Investigación en Dinámica Molecular, UAEM, Cuernavaca, Morelos, Mexico; \\ ${ }^{2}$ Instituto de Biotecnología, UNAM, Cuernavaca, Morelos, Mexico; \\ liz.garciav@gmail.com
}

In recent years, the genome of microorganisms from various habitats, such as industrial waste areas, areas rich in vegetable oils or in soils contaminated with oil, has been analyzed. This has allowed us to identify enzymes with functions that offer enormous potential for various applications in the industrial sector, from catalysis to remediation. Much of this knowledge has been leveled by bacteria of the genus Pseudomonas, since their metabolic versatility has been involved in a large number of biotechnological applications. Lipases catalyze the hydrolysis of triacylglycerides whose products are fatty acids and glycerol. These enzymes have a catalytic triad consisting of a serine, an acid residue (glutamic acid or aspartic acid) and a histidine. In addition, lipases have a preserved structure known as a lid. This lid is a mobile element that discovers the active site. It has been observed that lipase 2 (lip2) of Pseudomonas alcaligenes has a sequence identity of $48 \%$ with the lipase of $P$. aeruginosa, while the region of the lid has high identity with lids described in other halophilic or psychrophilic bacteria such as Marinobacter mobilis, Oleiphilus messinensis, Oleispira antartica or Hahellaceae bacterium, which makes it different from other lipases described until now. The lip 2 gene of $P$. alcaligenes was cloned into the Pet 28a vector and was expressed in Escherichia coli BL21 cells in order to obtain a crystallographic structure that allows us to describe and characterize the possible structural changes of the enzyme, as well as the possible implications of these changes in the stability and catalysis of lip 2 of $P$. alcaligenes.

Keywords: Lipase, enzime, lid 